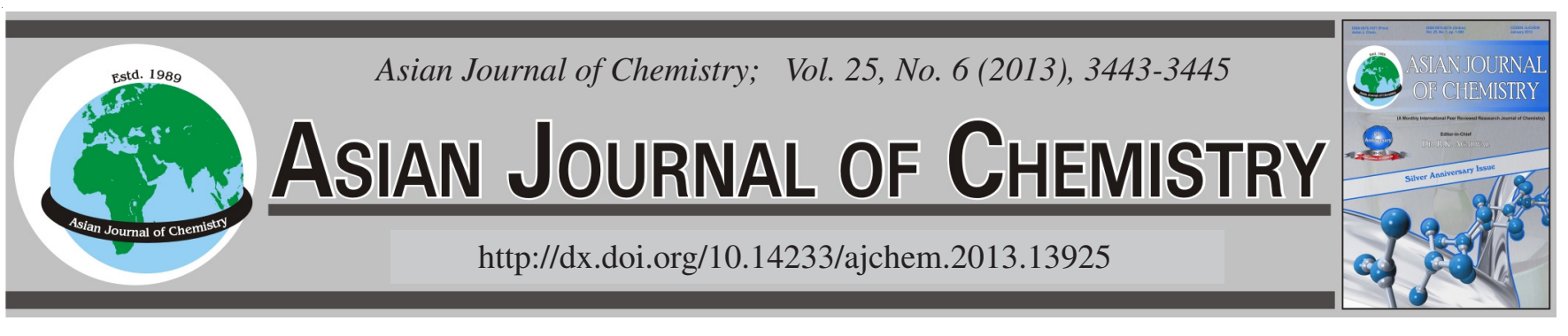

\title{
Synthesis and Supramolecular Structure of $2,2^{\prime}-\left\{1,1^{\prime}-[\right.$ Octane-1,8-diylbis(oxynitrilo)]diethylidyne $\}$ dinaphthol
}

\author{
Xiu-Yan Dong ${ }^{*}$, Li Wang, ChaO-Jing Zeng, FA WAng and YuAn Li
}

School of Chemical and Biological Engineering, Lanzhou Jiaotong University, Lanzhou 730070, P.R. China

*Corresponding author: E-mail: dxy568@163.com

(Received: 18 April 2012;

Accepted: 24 December 2012)

AJC-12610

The molecule of the title compound, $\mathrm{C}_{32} \mathrm{H}_{36} \mathrm{~N}_{2} \mathrm{O}_{4}$, assumes an E configuration with the dihedral angle between the two naphthalene rings of the molecule being $5.95(3)^{\circ}$. Two fairly strong intramolecular $\mathrm{O}-\mathrm{H} \cdots \mathrm{N}$ hydrogen bonds may, in part, influence the molecular conformation.

Key Words: Bisoxime compound, Synthesis, Crystal structure.

\section{INTRODUCTION}

Salen-type compounds are one of the most prevalent ligands in the field of coordination chemistry ${ }^{1}$. The development of their bisoxime analogues and complexes can provide new topologies for functional materials, in which coordination forms and functionality are important variables ${ }^{2}$. And can be used to obtain non-linear optical materials ${ }^{3}$, biological systems ${ }^{4}$, interesting magnetic properties ${ }^{5}$ and building blocks for cyclic supramolecular structures ${ }^{6}$. Thus, new materials can be produced by using these compounds, which seem to be suitable candidates for further chemical modifications ${ }^{7}$. Herein, we report on the synthesis and crystal structure of 2,2'-\{1,1'-[octane-1,8diylbis(oxynitrilo)]diethylidyne $\}$ dinaphthol.

\section{EXPERIMENTAL}

2-Acetyl-1-naphthol was purchased from Alfa Aesar and used without further purification. 1,8-Bis(aminooxy)octane was synthesized according to an analogous method reported earlier ${ }^{8}$. The other reagents and solvents were analytical grade reagents from Tianjin Chemical Reagent Factory. C, H and N analyses were carried out with a GmbH VariuoEL V3.00 automatic elemental analyzer. IR Spectra in the range 4000$400 \mathrm{~cm}^{-1}$ were recorded on a VERTEX70 FT-IR spectrophotometer using $\mathrm{KBr}$ pellets. The ${ }^{1} \mathrm{H}$ NMR spectra were recorded on a Mercury-400BB spectrometer at room temperature using $\mathrm{CDCl}_{3}$ as solvent. X-Ray single crystal structure was determined on a Bruker Smart 1000 CCD area detector. Melting points were measured by the use of a microscopic melting point apparatus made in Beijing Taike Instrument Limited Company and the thermometer was uncorrected.
General procedure: $2,2^{\prime}-\left\{1,1^{\prime}\right.$-[Octane-1,8-diylbis(oxynitrilo)]diethylidyne $\}$ - dinaphthol was synthesized according to an analogous method reported earlier ${ }^{9}$. To an ethanol solution $(5 \mathrm{~mL})$ of 2-acetyl-1-naphthol $(368.1 \mathrm{mg}$, $2.07 \mathrm{mmol}$ ) was added an ethanol solution $(3 \mathrm{~mL})$ of 1,8 bis(aminooxy)octane $(171.8 \mathrm{mg}, 0.97 \mathrm{mmol})$. The reaction mixture was stirred at $328-333 \mathrm{~K}$ for $70 \mathrm{~h}$. The formed precipitate was separated by filtration and washed successively with ethanol, respectively. The product was dried under vacuum to yield $374.4 \mathrm{mg}$ of the title compound. Yield, 75.3 \%. m.p. 392-394 K. Anal. calcd. (\%) for $\mathrm{C}_{32} \mathrm{H}_{36} \mathrm{~N}_{2} \mathrm{O}_{4}$ : C, 74.97; H, 7.08; N, 5.46. Found (\%): C, 74.95; H, 7.12; N, 5.40.

Colourless needle-like single crystals suitable for $\mathrm{X}$-ray diffraction studies were obtained after two months by slow evaporation from a ethanol/dichloromethane $(1: 1)$ mixed solution of the title compound.

X-Ray structure determination: The single crystal of the title compound, with approximate dimensions of 0.43 $\mathrm{mm} \times 0.19 \mathrm{~mm} \times 0.14 \mathrm{~mm}$ was placed on a Bruker Smart 1000 diffractmeter equipped with Apex CCD area detector. The diffraction data were collected using a graphite monochromated $\mathrm{MoK}_{\alpha}$ radition $(\lambda=0.71073 \AA)$ at 298(2) $\mathrm{K}$. The structure was solved by using the program SHELXS97 and Fourier difference techniques and refined by fullmatrix least-squares method on $\mathrm{F}^{2}$ using SHELXL-97. Details of the data collection and refinements of title compound are given in Table-1. The non-hydrogen atoms were refined anisotropically. Hydrogen atoms were added theoretically. CCDC: 875944. 


\begin{tabular}{ll}
\hline \multicolumn{1}{c}{ CRYSTAL DATA AND STRUCTURE } \\
\multicolumn{1}{c}{ REFINEMENT FOR THE TITLE COMPOUND } \\
\hline Empirical formula & $\mathrm{C}_{32} \mathrm{H}_{36} \mathrm{~N}_{2} \mathrm{O}_{4}$ \\
Formula weight & 512.63 \\
Temperature & $298(2) \mathrm{K}$ \\
Wavelength & $0.71073 \AA$ \\
Crystal system & Orthorhombic \\
Space group & Pca2(1) \\
Cell dimensions & $\mathrm{a}=23.140(3) \AA, \mathrm{b}=7.8910(10) \AA, \mathrm{c}$ \\
Volume & $=15.3369(18) \AA$ \\
$\mathrm{Z}$ & $2800.5(6) \AA^{3}$ \\
Density (calculated) & 4 \\
Absorption coefficient & $1.216 \mathrm{mg}^{3} \mathrm{~m}^{3}$ \\
$\mathrm{~F}_{\text {(000) }}$ & $0.080 \mathrm{~mm}^{-1}$ \\
Index ranges & 1096 \\
Reflections collected/unique & $-26 \leq \mathrm{h} \leq 27,-9 \leq \mathrm{k} \leq 9,-18 \leq 1 \leq 14$ \\
Independent reflections & $13599 / 3386\left[\mathrm{R}_{\text {(int) }}=0.0944\right]$ \\
Data/restraints/parameters & 938 \\
Goodness of fit indicator & $3386 / 1 / 345$ \\
R [I $>$ 26(I)] & 0.981 \\
Largest diff. peak and hole & $\mathrm{R}_{1}=0.0527, \mathrm{wR}_{2}=0.0820$ \\
\hline
\end{tabular}

\section{RESULTS AND DISCUSSION}

X-Ray crystallographic analysis revealed the crystal structure of title complex. The structure is shown in Fig. 1 while the packing arrangement of the unit cell is given in Fig. 2.

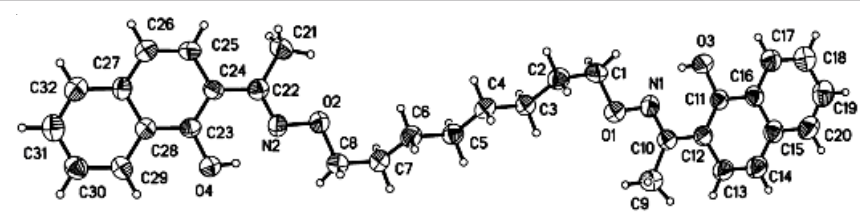

Fig. 1. Molecule structure of the title compound with atom numbering scheme. Displacement ellipsoids for non-hydrogen atoms are drawn at the $30 \%$ probability level

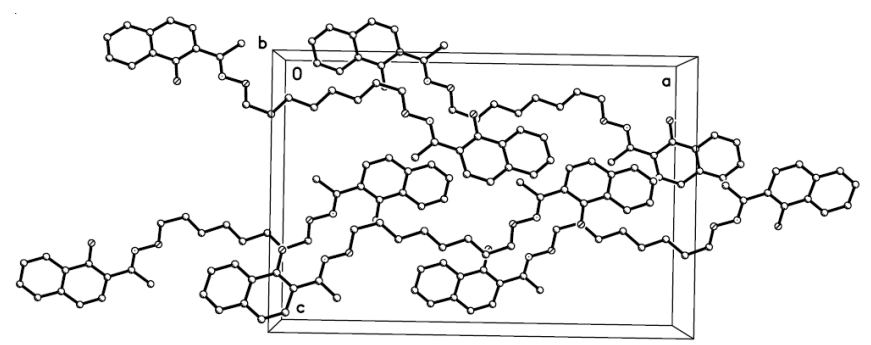

Fig. 2. Packing arrangement of the unit cell of the title compound

Selected bond distances and angles are listed in Table-2. The single crystal structure of the title compound is built up by only the $\mathrm{C}_{32} \mathrm{H}_{36} \mathrm{~N}_{2} \mathrm{O}_{4}$ molecule. The title compound is a typical Salen-type derivative with normal geometric parameters. The two pendant moieties attached to the ends of the -O- $(\mathrm{CH})_{8}-\mathrm{O}-$ backbone adopt an E conformation. The dihedral angle formed by the two naphthalene rings in each molecule is $c a .5 .95(3)^{\circ}$.

TABLE-2

SELECTED BOND DISTANCES $(\AA)$ AND ANGLES $\left({ }^{\circ}\right)$ FOR THE TITLE COMPOUND

\begin{tabular}{|c|c|c|c|c|c|}
\hline Bond & Lengths & Bond & Lengths & Bond & Lengths \\
\hline N(1)-C(10) & $1.284(7)$ & $C(7)-C(8)$ & $1.492(6)$ & $\mathrm{C}(21)-\mathrm{C}(22)$ & $1.510(7)$ \\
\hline $\mathrm{N}(1)-\mathrm{O}(1)$ & $1.414(5)$ & $\mathrm{C}(9)-\mathrm{C}(10)$ & $1.498(8)$ & $\mathrm{C}(22)-\mathrm{C}(24)$ & $1.442(8)$ \\
\hline $\mathrm{N}(2)-\mathrm{C}(22)$ & $1.300(7)$ & $\mathrm{C}(10)-\mathrm{C}(12)$ & $1.464(8)$ & $\mathrm{C}(23)-\mathrm{C}(24)$ & $1.389(7)$ \\
\hline $\mathrm{N}(2)-\mathrm{O}(2)$ & $1.390(5)$ & $\mathrm{C}(11)-\mathrm{C}(12)$ & $1.371(7)$ & $\mathrm{C}(23)-\mathrm{C}(28)$ & $1.395(7)$ \\
\hline $\mathrm{O}(1)-\mathrm{C}(1)$ & $1.420(6)$ & $\mathrm{C}(11)-\mathrm{C}(16)$ & $1.416(7)$ & $\mathrm{C}(24)-\mathrm{C}(25)$ & $1.412(8)$ \\
\hline $\mathrm{O}(2)-\mathrm{C}(8)$ & $1.444(6)$ & $\mathrm{C}(12)-\mathrm{C}(13)$ & $1.417(9)$ & $\mathrm{C}(25)-\mathrm{C}(26)$ & $1.356(6)$ \\
\hline $\mathrm{O}(3)-\mathrm{C}(11)$ & $1.365(6)$ & $\mathrm{C}(13)-\mathrm{C}(14)$ & $1.355(7)$ & $\mathrm{C}(26)-\mathrm{C}(27)$ & $1.388(7)$ \\
\hline $\mathrm{O}(4)-\mathrm{C}(23)$ & $1.353(6)$ & $\mathrm{C}(14)-\mathrm{C}(15)$ & $1.376(8)$ & $\mathrm{C}(27)-\mathrm{C}(32)$ & $1.390(8)$ \\
\hline $\mathrm{C}(1)-\mathrm{C}(2)$ & $1.489(6)$ & $\mathrm{C}(15)-\mathrm{C}(20)$ & $1.423(9)$ & $\mathrm{C}(27)-\mathrm{C}(28)$ & $1.421(8)$ \\
\hline $\mathrm{C}(2)-\mathrm{C}(3)$ & $1.508(6)$ & $C(15)-C(16)$ & $1.426(8)$ & $\mathrm{C}(28)-\mathrm{C}(29)$ & $1.410(7)$ \\
\hline$C(3)-C(4)$ & $1.518(5)$ & $\mathrm{C}(16)-\mathrm{C}(17)$ & $1.389(7)$ & $\mathrm{C}(29)-\mathrm{C}(30)$ & $1.374(6)$ \\
\hline$C(4)-C(5)$ & $1.505(5)$ & $\mathrm{C}(17)-\mathrm{C}(18)$ & $1.364(7)$ & $\mathrm{C}(30)-\mathrm{C}(31)$ & $1.381(8)$ \\
\hline$C(5)-C(6)$ & $1.504(5)$ & $\mathrm{C}(18)-\mathrm{C}(19)$ & $1.406(8)$ & $\mathrm{C}(31)-\mathrm{C}(32)$ & $1.367(7)$ \\
\hline $\mathrm{C}(6)-\mathrm{C}(7)$ & $1.505(6)$ & $\mathrm{C}(19)-\mathrm{C}(20)$ & $1.333(9)$ & - & - \\
\hline $\mathrm{C}(10)-\mathrm{N}(1)-\mathrm{O}(1)$ & $113.3(5)$ & $\mathrm{C}(11)-\mathrm{C}(12)-\mathrm{C}(13)$ & $116.9(6)$ & $\mathrm{O}(4)-\mathrm{C}(23)-\mathrm{C}(24)$ & $121.9(6)$ \\
\hline $\mathrm{C}(22)-\mathrm{N}(2)-\mathrm{O}(2)$ & $113.6(5)$ & $\mathrm{C}(11)-\mathrm{C}(12)-\mathrm{C}(10)$ & $123.1(7)$ & $\mathrm{O}(4)-\mathrm{C}(23)-\mathrm{C}(28)$ & $115.2(6)$ \\
\hline $\mathrm{N}(1)-\mathrm{O}(1)-\mathrm{C}(1)$ & $108.8(5)$ & $C(13)-C(12)-C(10)$ & $120.0(7)$ & $\mathrm{C}(24)-\mathrm{C}(23)-\mathrm{C}(28)$ & $122.9(6)$ \\
\hline $\mathrm{N}(2)-\mathrm{O}(2)-\mathrm{C}(8)$ & $108.0(4)$ & $\mathrm{C}(14)-\mathrm{C}(13)-\mathrm{C}(12)$ & $121.0(7)$ & $\mathrm{C}(23)-\mathrm{C}(24)-\mathrm{C}(25)$ & $117.2(6)$ \\
\hline $\mathrm{O}(1)-\mathrm{C}(1)-\mathrm{C}(2)$ & $108.1(5)$ & $\mathrm{C}(13)-\mathrm{C}(14)-\mathrm{C}(15)$ & $122.8(8)$ & $\mathrm{C}(23)-\mathrm{C}(24)-\mathrm{C}(22)$ & $122.8(6)$ \\
\hline$C(1)-C(2)-C(3)$ & $114.9(5)$ & $\mathrm{C}(14)-\mathrm{C}(15)-\mathrm{C}(20)$ & $123.8(9)$ & $\mathrm{C}(25)-\mathrm{C}(24)-\mathrm{C}(22)$ & $119.9(6)$ \\
\hline $\mathrm{C}(2)-\mathrm{C}(3)-\mathrm{C}(4)$ & $113.7(5)$ & $\mathrm{C}(14)-\mathrm{C}(15)-\mathrm{C}(16)$ & $118.4(7)$ & $\mathrm{C}(26)-\mathrm{C}(25)-\mathrm{C}(24)$ & $120.4(6)$ \\
\hline $\mathrm{C}(5)-\mathrm{C}(4)-\mathrm{C}(3)$ & $113.4(4)$ & $\mathrm{C}(20)-\mathrm{C}(15)-\mathrm{C}(16)$ & $117.9(8)$ & $\mathrm{C}(25)-\mathrm{C}(26)-\mathrm{C}(27)$ & $123.2(6)$ \\
\hline$C(6)-C(5)-C(4)$ & $114.2(4)$ & $\mathrm{C}(17)-\mathrm{C}(16)-\mathrm{C}(11)$ & $123.6(8)$ & $\mathrm{C}(26)-\mathrm{C}(27)-\mathrm{C}(32)$ & $123.8(7)$ \\
\hline$C(5)-C(6)-C(7)$ & $114.3(5)$ & $\mathrm{C}(17)-\mathrm{C}(16)-\mathrm{C}(15)$ & $118.7(7)$ & $\mathrm{C}(26)-\mathrm{C}(27)-\mathrm{C}(28)$ & $117.7(6)$ \\
\hline$C(8)-C(7)-C(6)$ & $113.4(5)$ & $\mathrm{C}(11)-\mathrm{C}(16)-\mathrm{C}(15)$ & $117.6(7)$ & $\mathrm{C}(32)-\mathrm{C}(27)-\mathrm{C}(28)$ & $118.5(7)$ \\
\hline $\mathrm{O}(2)-\mathrm{C}(8)-\mathrm{C}(7)$ & $108.3(5)$ & $\mathrm{C}(18)-\mathrm{C}(17)-\mathrm{C}(16)$ & $121.6(7)$ & $\mathrm{C}(23)-\mathrm{C}(28)-\mathrm{C}(29)$ & $122.6(6)$ \\
\hline $\mathrm{N}(1)-\mathrm{C}(10)-\mathrm{C}(12)$ & $116.0(7)$ & $\mathrm{C}(17)-\mathrm{C}(18)-\mathrm{C}(19)$ & $119.8(7)$ & $\mathrm{C}(23)-\mathrm{C}(28)-\mathrm{C}(27)$ & 118.6(6) \\
\hline $\mathrm{N}(1)-\mathrm{C}(10)-\mathrm{C}(9)$ & $122.3(6)$ & $\mathrm{C}(20)-\mathrm{C}(19)-\mathrm{C}(18)$ & $120.4(8)$ & $\mathrm{C}(29)-\mathrm{C}(28)-\mathrm{C}(27)$ & 118.7(6) \\
\hline $\mathrm{C}(12)-\mathrm{C}(10)-\mathrm{C}(9)$ & $121.7(7)$ & $\mathrm{C}(19)-\mathrm{C}(20)-\mathrm{C}(15)$ & $121.6(8)$ & $\mathrm{C}(30)-\mathrm{C}(29)-\mathrm{C}(28)$ & $120.7(6)$ \\
\hline $\mathrm{C}(12)-\mathrm{C}(11)-\mathrm{C}(16)$ & $123.2(6)$ & $\mathrm{C}(24)-\mathrm{C}(22)-\mathrm{C}(21)$ & $121.5(6)$ & $\mathrm{C}(31)-\mathrm{C}(32)-\mathrm{C}(27)$ & $121.5(7)$ \\
\hline
\end{tabular}


This structure is different from what was observed in our previously reported Salen-type bisoxime compounds ${ }^{9-12}$, due to the carbon chain of title compound is longer than of them. Two intramolecular hydrogen bonds, $\mathrm{O} 3-\mathrm{H} \cdots \mathrm{N} 1$ and $\mathrm{O} 4-$

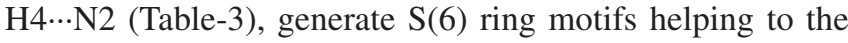
stabilization of the title molecule ${ }^{13-15}$.

TABLE-3

HYDROGEN BONDS $\left[\AA{ }^{\circ},{ }^{\circ}\right.$ FOR THE TITLE COMPOUND

\begin{tabular}{ccccc} 
D-H $\cdots \mathrm{A}$ & $\mathrm{d}(\mathrm{D}-\mathrm{H})$ & $\mathrm{d}(\mathrm{H} \cdots \mathrm{A})$ & $\angle \mathrm{DHA}$ & $\mathrm{d}(\mathrm{D} \cdots \mathrm{A})$ \\
\hline $\mathrm{O} 3-\mathrm{H} 3 \cdots \mathrm{N} 1$ & 0.82 & 1.84 & 144 & $2.548(3)$ \\
$\mathrm{O} 4-\mathrm{H} 4 \cdots \mathrm{N} 2$ & 0.82 & 1.81 & 146 & $2.528(2)$ \\
\hline
\end{tabular}

\section{ACKNOWLEDGEMENTS}

The authors acknowledge finanical support from the 'Jing Lan' Talent Engineering Funds of Lanzhou Jiaotong University.

\section{REFERENCES}

1. S. Akine, T. Taniguchi and T. Nabeshima, Angew. Chem. Int. Ed., 41, 4670 (2002)
2. W.K. Dong, G. Wang, S.S. Gong, J.F. Tong, Y.X. Sun and X.H. Gao, Transition Met. Chem., 37, 271 (2012).

3. H. Miyasaka, N. Matsumoto, H. Okawa, N. Re, E. Gallo and C. Floriani, J. Am. Chem. Soc., 118, 981 (1996).

4. P.G. Lacroix, Eur. J. Inorg. Chem., 339 (2001).

5. T.K. Ronson, H. Adams and M.D. Ward, Inorg. Chim. Acta, 358, 1943 (2005).

6. A.K. Sharma, F. Lloret and R. Mukherjee, Inorg. Chem., 46, 5128 (2007).

7. C. Policar, F. Lambert, M. Cesario and I. Morgenstern-Badarau, Eur. J. Inorg. Chem., 2201 (1999).

8. D.A. Atwood and M.J. Harvey, Chem. Rev., 101, 37 (2001).

9. W.K. Dong, X.N. He, H.B. Yan, Z.W. Lv, X. Chen, C.Y. Zhao and X.L. Tang, Polyhedron, 28, 1419 (2009).

10. W.K. Dong and J.H. Feng, Acta Cryst., E62, o3577 (2006).

11. W.K. Dong, J.H. Feng and X.Q. Yang, Z. Kristallogr. New Cryst. Struct., 221, 447 (2006).

12. W.K. Dong, J.Y. Shi, Y.X. Sun, L. Wang, J.G. Duan, J.K. Zhong and L. Xu, Anal. Sci., 23, x167 (2007)

13. H.L. Wu, X.C. Huang, J.K. Yuan, F. Kou, F. Jia, B. Liu and Y. Bai, Z. Naturforsch, 66b, 1049 (2011).

14. W.K. Dong, Y.X. Sun, Y.P. Zhang, L. Li, X.N. He and X.L. Tang, Inorg. Chim. Acta, 362, 117 (2009).

15. W.K. Dong, J.G. Duan, Y.H. Guan, J.Y. Shi and C.Y. Zhao, Inorg. Chim. Acta, 362, 1129 (2009). 